\title{
Advances in Thin Film Techniques for Optical, Microwave and Millimeter wave Circuit Applications
}

\author{
K.Callery ${ }^{1}$ \& P.Lowbridge ${ }^{2}$ \\ 1. Aeroflex MIC Technology Corporation, 100 Andover By Pass, North Andover, MA 01845, USA \\ Tel 001-978-687-9625 Fax 001-978-687-9721 E-mail: kcallery@mic.aeroflex.com \\ 2. Novacom Microwave Ltd, Unit 6, 22 The Green, Nettleham, Lincoln, LN2 2NR, England, UK \\ Tel +44 1522751136 Fax +44 1522754408 E-Mail: paul.lowbridge@novacom-mwave.com
}

\section{INTRODUCTION}

In this paper, the high volume process (as developed by Aeroflex MIC Technology) for depositing thin films comprising TaN, Ti, Ti/Pd alloy, $\mathrm{Cu}, \mathrm{Ni}, \mathrm{Au}$ (TTPCNA) onto materials such as alumina and aluminium nitride is presented. The benefits of this tested methodology are realized by improved process control, a reduction in cycle time, and minimal operator intervention in the process. These important considerations in today's competitive manufacturing environment translate into higher yields, reduced cost to the customer and reliability in the field. This high volume line has a current run rate in excess of 24,000 substrates per week as it is presently configured with the ability to expand to 35,000 substrates per week with minimal investment. We then present a case study of the application of this technology, together with the ability to pre-deposit gold-tin solder, in the exacting application of mounting Laser diodes onto thin film heatsinks - which also convey the microwave / millimeter wave signal.

\section{OVERVIEW OF THE HIGH VOLUME PROCESS}

The Aeroflex MIC Technology high-volume manufacturing platform is based on continuous flow manufacturing processes. To insure the highest quality as well as highest throughput, precision equipment control parameters have been designed into the individual tools. Automated cassette to cassette material handling technology expedites substrate transfers and reduces breakage during processing. The standard substrate size for processing is $3.75 "(92.25 \mathrm{~mm}) \times$ x $4.50 "(114.30 \mathrm{~mm})$.

\subsection{Basic Processes}

There are seven basic processes that make up the high volume production platform.

\subsubsection{Thick film deposition}

Automated thick film screen printers are employed to deposit the gold ink to form the conductive element of the crossunder structures. The thick film gold is printed in a single pass, and can resolve features of 150 microns after firing. Subsequently, the insulator layer is deposited through two print and fire cycles. The double application of the glaze insulator insures complete isolation. The resulting composite structure has a thickness of 40 microns.

\subsubsection{Sputtering}

Interconnection to the crossunder structure is accomplished with either a resistor of conductor, produced from a thin film metalization. The metalization used is $\mathrm{Ti}, \mathrm{Ti} / \mathrm{Pd}$ alloy and $\mathrm{Cu}$ for all conductors; sputtering $\mathrm{TaN}$ prior to the conductor layers permits formation of the resistors. This highly reliable structure has been used as a standard for communication and microwave interconnect circuits for more than 25 years.

The sputtering equipment platform consists of six systems, two for resistor and four for conductor deposition. Each system is identical in layout except for cathode material and placement. Many optimization concepts were designed into the systems to maximize efficiency.

- Maximization of substrate area with a cylindrical, peripheral substrate arrangement.

- Separately pumped loading and unloading air lock.

- Large capacity magazine transfer through the air lock.

- Protection of and compensation for variations in substrates by use of picture frame type carriers.

- Vertical positioning of substrates and cathode, Sixteen cathodes positions (eight per side).

- Aperture shielding of thickness uniformity.

- Distributed reactive gas for chemical composition uniformity.

The throughput of the sputtering platform is unparalleled in thin film interconnect manufacturing with each resistor system being capable of cycling 750 substrates per hour, and the conductor system cycling 350 substrates per hour.

The (simultaneous) two-sided deposition allows for homogeneous through-hole coating, without the intermetallic problems that are unavoidable with conventional down or side sputter systems. 
One extra feature, which this system also enables is the realization of solid copper filled vias. This is a patented technique and enables solid (pure) copper vias to be used at strategic positions in microstrip circuits for enabling such features as heat sinking and solid conductive grounding.

\subsubsection{Conductor photolithography}

Crossunder technology and the typical 6 to 12 micron conductor thickness' associated with thin film interconnects require conformal resist applications. Aeroflex MIC Technology utilizes a proprietary electrophoretic photo polymer to generate the conductor pattern. This proprietary photoresist formulation provides crisp vertical side walls and line resolution reliably down to 25 microns. By plating the photoresist onto the substrate, step coverage is consistent and predictable at 12 to 21 microns in thickness. The photoresist plating system is fully automated using single substrate Continuous Substrate Flow (CSF) with a capacity off 200 substrates per hour. The electrophoretic bath parameters are controlled through a proprietary process advisor system that statistically monitors key parameters.

Photoresist exposure is performed on six projection exposure towers, through 1:1 chrome photo tools with pellicles. Three of the towers are configured for dual sided exposure, allowing for simultaneous front and back exposure of the substrate. Their capacity is 100 substrates per hour per unit. The other three units are configured with single light sources and carousel cassette loaders. These are single sided exposure systems, and each has a capacity of 180 substrates per hour. All systems allow for either precision optical or hard tool alignment as required by the complexity of the circuits being exposed.

Developing the photoresist after the exposure is accomplished using a CSF processor with computerized temperature monitoring and speed adjustment. This system has a capacity of 600 substrates per hour.

\subsubsection{Conductor pattern plating}

Conductor film thickness is increased by selectively electroplating copper, nickel and gold. The plating cells are designed to hold one process lot of material. The fixture, when loaded into the cell, creates an anode to cathode spacing of less than $10 \mathrm{~mm}$ with a greater than $1: 1$ ratio, anode to cathode. In addition, the configuration of the box plater allows for liquid flows of 55 to 65 liters per minute within the plating cell. This high rate of fluid exchange between the anode and cathode creates a non-depleting zone from which much higher than traditional plating rates are attained without sacrificing uniformity. Aeroflex MIC Technology currently has two complete plating lines, with a total capacity of 240 substrates per hour.

The conductor seed metals are removed down to either the substrate or the resistor layer by spray etching. The high volume spray etcher is configured to sequentially remove each metal layer with rinse steps in between to eliminate solution contamination. The system has been designed as a CSF processor with a capacity of 450 substrates per hour.

\subsubsection{Resistor photolithography}

Resistor features are formed using sprayed photo resist. The resist sprayer is self contained and configured with cassette to cassette loaders, providing capacity in excess of 600 substrates per hour. Five to six microns of resist is sprayed in the main chamber and dried with radiant heat. After the belt delivers the substrates to the download cassettes, the belt is cleaned and dried prior to returning to the load end of the spray booth.

Resistor etching is performed in a batch mode due to the rapid rate at which tantalum nitride films can be etched. The substrates are loaded into a process carrier and manually placed into the etch solution. When all the metal is removed, they are transferred to an active counter flow rinse system, equipped with agitation. The capacity of this process cell is in excess of 500 substrates per hour.

\subsubsection{Laser resistor trimming}

Resistor trimming is accomplished with YAG Laser systems. Ten ESI brand trimmer systems are used in production for all standard products. Two Teradyne Laser systems are utilized for any specific requirements of engineering. The standard production systems have a resolution of 30 milliohms, and are capable of performing all common resistor cuts. Standard resistor tolerances offered range from $1 \%$ to $20 \%$. Resistors can be trimmed to a tolerance of less than $1 \%$, with a substantial increase in cost.

\subsubsection{Sizing of individual die}

Separation of die is accomplished either through precision diamond saws or $\mathrm{CO} 2$ Lasers. The precision diamond saws utilize micro grinding wheels of up to $125 \mathrm{~mm}$ diameter that increase accuracy and throughput. Typical dicing tolerances are $+/-0.05 \mathrm{~mm}$ with capability down to $+/-0.013 \mathrm{~mm}$ at an increased cost. CO2 Laser separation of die is the most economical alternative with typical tolerances from $0.13 \mathrm{~mm}$ to $0.23 \mathrm{~mm}$. Typically, Laser scribing is performed on the circuit side of the substrate. Backside scribing can also be performed, if appropriate alignment targets are planned for.

\subsection{Key Attributes of the process}

The key parameters and achievable physical geometries of this high volume process are described in 
Table 1 . The result is a high volume process optimized for cost, reliability and repeatability and ideally suited for realizing optical, microwave and millimeter wave thin film circuits.

\section{CASE STUDY - LASER DIODE MOUNTING USING PRE-DEPOSITED AUSn SOLDER}

Many obstacles have been encountered in applications where Laser diodes need to be mounted so that the radiating edge is coincident with the edge of the substrate (see Figure A). The available high volume substrate manufacturing methods have in some cases resulted in decreased optical efficiency or increased thermal resistance. These undesirable conditions are caused by a lack of parallelism of the diode to the substrate or unsupported diode surface area.

Cost efficient high volume manufacturing practices require that substrate interconnects be processed in an array format. Therefore, to have metalization terminate at the substrate edge requires patterned metal to extend into the kerf area between the arrayed die. The extended metal subsequently is cut through when separating the array. The blades used on dicing saws are specifically designed to cut (grind) through hard polycrystalline and crystalline materials and when passing through soft materials like Au and Cu a burr is created in the metal at the diced edge. Unless the burrs are manually removed prior to assembly, the radiating edge of the attached diode can be elevated by the burr, resulting in misalignment of the optical system and the creation of solder voids which could result in a loss of both optical and thermal efficiency.

\subsection{Potential Solution using Pull-Back techniques}

One design option for "P" up configuration Laser diodes assemblies is to pull back the metalization from the edge (typically .002"). This design requires alignment of the Laser diode to the edge of the metal platform or increased thermal resistance can result at the unsupported portion of the diode (see Figure B). Pulled back placement in "P" down configurations is often not an acceptable solution due to an obstructed light path translating to a loss in optical efficiency. Additional alternatives for pulled back placement are pedestals made of high thermally conductive material, or ground $45^{\circ}$ chamfers at the substrate edge so that the light emitting from the diode is not clipped. Pedestals have the added cost associated with one additional Au/Sn preform, the pedestal itself as well as the increased assembly cost including the associated yield loss. In general, these alternatives hamper the ability to produce in high volume competitively and add no additional value or performance advantages.

\subsection{Preferred Solution - using Pre-Deposited AuSn terminated at the circuit edge}

Aeroflex MIC Technology has worked with major OEM's to develop an integrated 80/20 Au/Sn eutectic deposition process that effectively addresses the concerns of edge placement and thermal management and provides a straightforward, cost effective solution. In addition, by integrating the eutectic solder $(80 / 20 \mathrm{Au} / \mathrm{Sn})$ into the base substrate, placement of eutectic preforms is eliminated. By utilizing thinner integrated solder layers (from 4-6 microns in thickness), overall thermal performance is improved compared to the 12 to 14 micron eutectic preforms normally utilized in these assemblies.

Aeroflex MIC Technology's eutectic solder coated diode platform terminates at the edge. This is accomplished through a multistage, additive process where the attachment pad topography is selectively tiered so that the area that approaches the edge is at a lower level than the balance of the pad (Figure C). The height difference, which begins less than.001" from the substrate edge, produces a controlled gap that fills with eutectic solder during reflow forming an integral solder joint to the edge (Figure D).

The integrated 80/20, Au/Sn eutectic solder is offered from 3 to 6 microns in thickness and can be patterned for multiple attachment sites. Eutectic freeze times (elapsed time from liquid phase back to solid crystalline phase) at a controlled temperature of $290^{\circ} \mathrm{C}$ are from 3 to 5 minutes depending on solder thickness.

\section{CONCLUSIONS}

The ability to efficiently manufacture high volume thin film circuits with fine features, integral solid filled vias, resistors and cross-unders opens up possibilities which were previously discounted due to the high price of such circuits. The high volume Optical market has benefited greatly from this technology - which has enabled the complex procedure of mounting the Laser diode onto an efficient heatsink, with the microwave / millimeter wave circuitry on the same tile, to become a standard and common place process. The ability to integrate many fine features at a low cost has also meant that thin film is now a preferred solution for several $\mathrm{RF}$ and microwave applications which were formerly the domain of softboard technologies.

\section{ACKNOWLEDGEMENTS}

The authors would like to acknowledge the inputs and support from colleagues at Aeroflex MIC Technology with especial thanks to Malcolm Hill for his technical input, and Christine Williams for coordinating the inputs. 
TABLE 1: Attributes of the Metalization scheme

\begin{tabular}{|c|c|c|}
\hline Substrate Material & \multicolumn{2}{|c|}{$\begin{array}{l}\text { Alumina }(99.5 \% \text { or } 99.6 \%) \\
\text { Aluminium nitride }\end{array}$} \\
\hline Metal Scheme: & $\begin{array}{ll}\mathrm{TaN} & \text { (sputtered) } \\
\mathrm{Ti} & \text { (sputtered) } \\
\mathrm{TiPd} & \text { (sputtered) } \\
\mathrm{Cu} & \text { (sputtered) } \\
\mathrm{Cu} & \text { (plated) } \\
\mathrm{Ni} & \text { (plated) } \\
\mathrm{Au} & \text { (plated) } \\
\mathrm{AuSn} & \text { (sputtered) }\end{array}$ & $\begin{array}{l}50 \text { / } 100 \text { ohms per square(optional) } \\
0.2 \mathrm{um} \\
0.1 \mathrm{um} \\
0.35 \mathrm{um} \\
3 \text { to } 8 \text { um (optional) } \\
1.0 \text { to } 3.0 \mathrm{um} \\
0.5 \text { to } 3.0 \mathrm{um} \\
3.0 \text { to } 6.0 \mathrm{um} \text { (optional) }\end{array}$ \\
\hline $\begin{array}{ll}\text { Features } \\
-\quad \text { lines \& spaces on cross-under(s) (thick film) } \\
- & \text { cross-over / interconnect } \\
- & \text { lines and spaces (thin film) } \\
- & \text { plated via diameter } \\
- & \text { solid (copper filled) filled via diameter }\end{array}$ & \multicolumn{2}{|l|}{$\begin{array}{l}\text { 150um (min) } \\
\text { 125um (min) } \\
\text { 40um (min) } \\
\text { 300um (min) } \\
\text { 300um (min) }\end{array}$} \\
\hline \begin{tabular}{ll}
\multicolumn{2}{l}{ Resistor Parameters } \\
- & TCR \\
- & Tolerance (trimmed) \\
- & Current density
\end{tabular} & \multicolumn{2}{|c|}{$\begin{array}{l}-90+/-50 p p m \text { per deg C } \\
1 \% \text { to } 20 \% \\
5 \times 10^{4} \text { amps per sq. } \mathrm{cm}\end{array}$} \\
\hline Thermo compression bondable & \multicolumn{2}{|l|}{ Yes } \\
\hline Solderable & \\
\hline
\end{tabular}

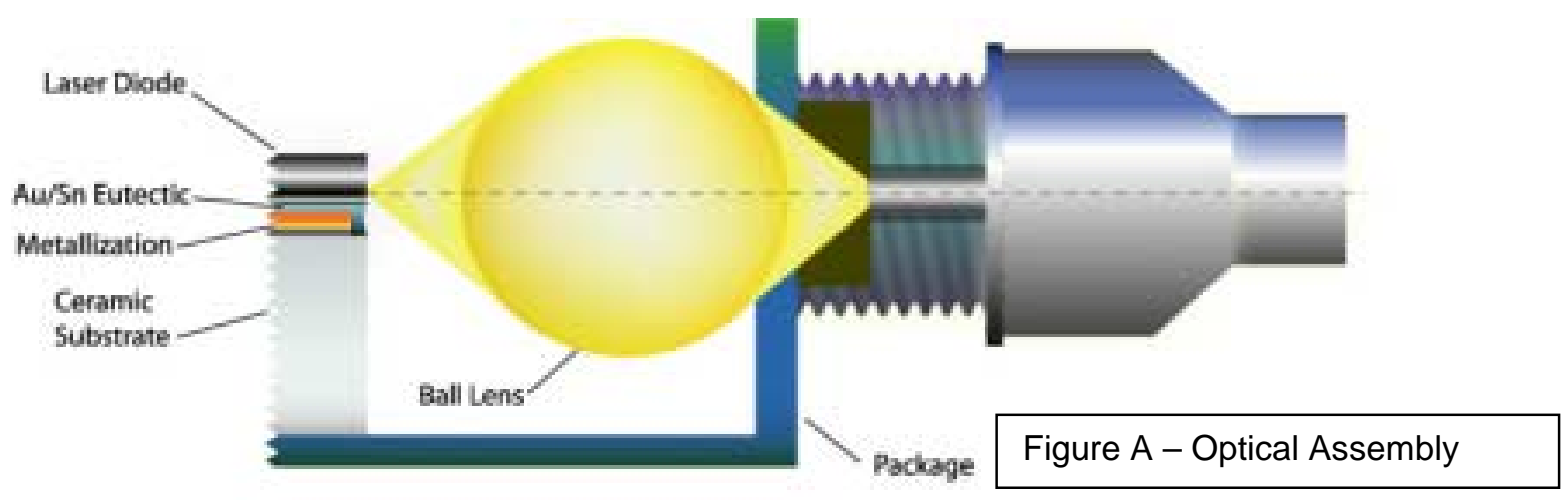

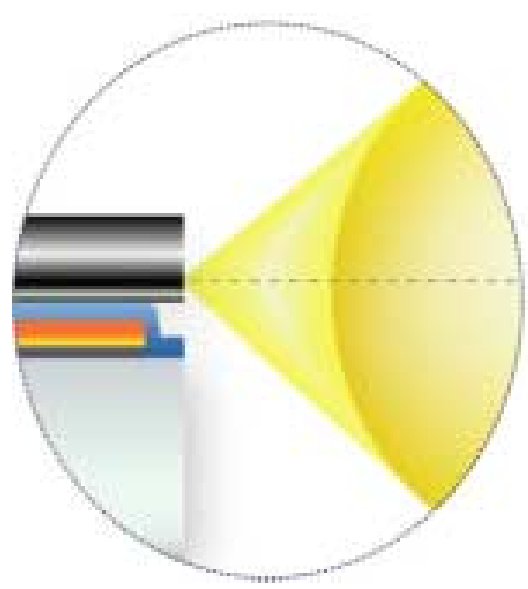

Figure $\mathrm{C}$ - Before reflow

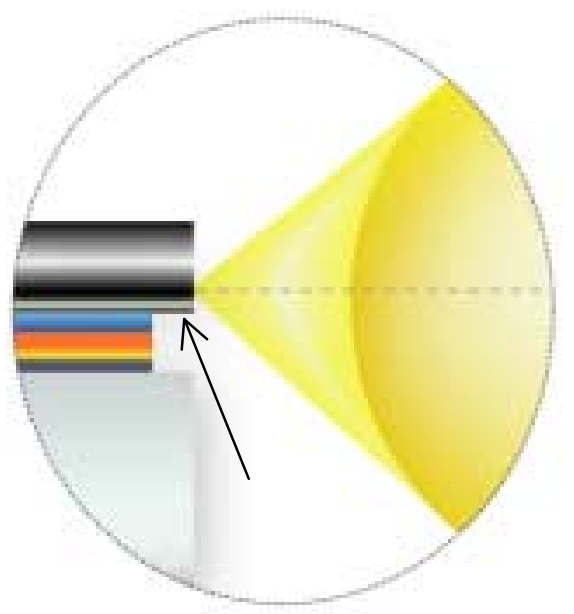

Figure $\mathrm{B}-$ with pull back

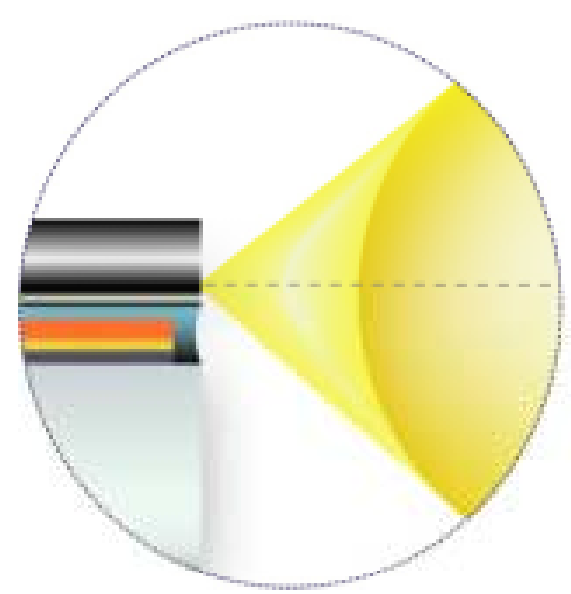

Figure D - After reflow 